\title{
Application of Multi-Attribute Decision Making Approach for Transesterification Process using Grey Relational Analysis
}

\author{
S. Balamurugan
}

Sri Krishna College of Engineering and Technolgy, Coimbatore, Tamil Nadu, India - 641008

Received 22 August 2019; Accepted 21 August 2020

\begin{abstract}
This study focuses on selection of optimum transesterification process parameters of linseed oil as a potential feedstock for bio-fuel production in the presence of calcium oxide $(\mathrm{CaO})$ obtained from the waste eggshells. The waste chicken eggshells were calcined at $900{ }^{\circ} \mathrm{C}$ for $4 \mathrm{~h}$ and it was characterised by X-ray diffractometer (XRD). The transesterification process was conducted according to L9 orthogonal array with selected input control parameters such as methanol to oil molar ratio, reaction temperature and catalyst loading. The output parameters measured are the biodiesel yield and viscosity. The multi-objective decision-making technique called Grey Relational Analysis (GRA) was used to identify the optimum transesterification process parameters to maximize the biodiesel yield and to minimize the biodiesel viscosity. The optimized values of transesterification process parameters are methanol to oil molar ratio of $8: 1$, a reaction temperature of $65^{\circ} \mathrm{C}$ and catalyst loading of $7 \% \mathrm{w} / \mathrm{w}$.
\end{abstract}

Keywords: Decision Makinh, Grey, Analysis

\section{Email: sambalamurugan@gmail.com}

\section{Introduction}

Fossil fuel plays an important role in the transportation sector. Continues usage of fossil fuel in the automobiles emits harmful gases like $\mathrm{CO}, \mathrm{NO}_{\mathrm{x}}, \mathrm{HC}$ and Sulphur content to the environment. Emission of these kinds of gases increases the temperature of the earth. Increasing in the temperature of earth leads to effect in the global warming [1]. In the other hand, continuous usage of fossil fuel reduces its availability. Concerning the fossil fuel availability and its harmful effects, gives rise to think of the importance of the bio-fuels [2].

Combustion of bio-fuel in the internal combustion engine reduces the global warming rate as this bio-fuel involves in the closed carbon cycle and hence reduces the green house gas emissions. Bio-fuels comprise of many benefits and the major advantages are eco-friendly, renewable and biodegradable and non-toxic. Bio-fuel has $12 \%$ of oxygen, this adds further advantage and this controls emissions in greater ranges [3].

Generally non-edible oil was used for bio-fuel preparation. Non-edible oil to bio-fuel conversion, transesterification process was used. Transesterification was an effective chemical process used to reduce the viscosity of the non-edible oil. Normally, non-edible oil is higher viscosity in nature and this high viscosity oil is not recommended to use directly in a compression ignition engine for combustion. High viscosity oil stick on the surface of injector nozzle as it forced to atomize. This leads to improper atomization which affects proper combustion [4].

In the transesterification process, non-edible oil along with alcohol and catalyst was properly mixed and heated for

*E-mail address: sambalamurugan@gmail.com

ISSN: $1791-2377$ @ 2020 School of Science, IHU. All rights reserved.

doi:10.25103/iestr.134.21 elevated temperature. As continuous transfer of heat, triglyceride of non-edible oil was converted into Fatty Acid Methyl Ester (FAME) and this FAME is termed as bio-fuel $[1]$.

Two category of catalyst are widely used in the transesterification process, namely homogeneous and heterogeneous catalyst. Homogeneous catalyst has its own drawbacks as multi washing of transesterified oil was required to remove catalyst residues and also produces unwanted waste water which then creates excess soap byproduct. Homogeneous catalyst was not reusable and corrosive to equipment. Heterogeneous catalyst overcomes homogeneous catalysts drawbacks. Homogeneous solid catalyst easy to recover and reusable for several times and excess waste water production was eliminated [5].

Generally, all catalyst produces its impact on environmental concerns. Therefore, development of solid catalyst has recently gained much attention among the researchers to overcome the aforementioned issues. In this regard a waste eggshell catalyst is a potential source for producing low cost bio-fuel. Waste eggshell contains high amount of calcium components $\left(\mathrm{CaCO}_{3}\right)$ and this $\mathrm{CaCO}_{3}$ could be easily converted to Calcium Oxide $(\mathrm{CaO})$ by calcinations method. Calcined $\mathrm{CaO}$ was a potential catalyst as it was cheap, non corrosive, eco-friendly and reusable [6].

Calcium oxide $(\mathrm{CaO})$ catalyst was prepared from waste chicken eggshell by calcinations process. Transmission electron microscope (TEM) image showed spherical structure with average particle size as $46.1 \pm 2.1 \mathrm{~mm}$. RSM was used to optimize the transesterification process parameters [7]. Calcination-hydration-dehydration technique was used to convert the waste eggshell into catalyst. TEM image showed that spherical shape with average particle size of $75 \mathrm{~nm}$. The effect of prepared catalyst was analysed in the 
transesterification process of dry biomass into biodiesel [4]. Ostrich-eggshell and chicken-eggshell were calcined and used for transesterification process. Waste cooking oil was converted as bio-fuel. RSM and Taguchi method was applied to optimize the process parameters. The optimum biodiesel yield of approximately $98 \%$ (ostrich eggshell) and 96\% (chicken eggshell) were achieved [8]. CaO catalyst was derived from waste shells of egg, oyster and clam. Transesterification of soybean oil was performed. Sonication was done on $\mathrm{CaO}$ catalyst about five hours which resulted in reduction of particle size by $34 \%$ and this resulted in a $56 \%$ increase in the activity [9]. A simple wet impregnation method was used to make solid bifunctional tungsten molybdenum supported calcium mixed oxide catalyst. The prepared catalyst was used in a transesterification of waste cooking oil. This catalyst was recorded the highest bio-fuel yield of $96.2 \%$ under optimized condition [5]. Simple calcinations process was used to convert eggshell into $\mathrm{CaO}$ catalyst at $900^{\circ} \mathrm{C}$ for 4 hours. Chicken oil was used in the synthesis of bio-fuel. RSM was used to optimize the transesterification parameters. Maximum biodiesel yield of $90.41 \%$ was recorded in this study [10]. The waste eggshell was calcined at $800^{\circ} \mathrm{C}$ and used in the transesterifiation of dairy industry waste scum oil. Maximum bio-fuel yield of 96 $\%$ was obtained at the optimized parameters [11]. An efficient catalyst was derived from the waste eggshell by calcination process. Date seed oil was used in transesterification process. $93.5 \%$ of bio-fuel at optimized condition was recorded in this study [12].

The literature provides knowledge that still there was a space to develop an effective and efficient catalyst for transesterification process to produce economical and ecofriendly bio-fuel in the future. Selection of feedstock for the bio-fuel synthesis plays major role in the economical aspects. Continuous availability of feedstock and catalyst derived from waste eggshell was the key area to produce low cost biofuel. Considering continuous availability, linseed oil was considered in this work for the bio-fuel synthesis process. This oil is non-edible and used in the paint manufacturing industries. This work aimed to produce cheap and effective catalyst from the waste chicken eggshell by calcination process and also from the literature reviews; it can be observed that many researchers have focused on optimizing single response problem in the transesterification process. A scanty of work carried out in the multi-objective optimization of transesterification experiments. In order to fill this gap, the present study uses Grey Relational Analysis (GRA) techniques to optimize the bio-fuel production from the linseed oil along with $\mathrm{CaO}$ catalyst prepared from the waste chicken eggshell.

\section{Materials and Methods}

\subsection{Materials}

Linseed oil was purchased from the nearby market. Linseed oil was extracted from the linseed by pre-expelling and hexane extraction of the press cake method. Oil is in yellowish. Linseed oil has better properties than diesel fuel. Fuel properties of linseed oil like viscosity, flash point, fire point, could point and pour point, densities are shown in the Table. 1[13].

\subsection{Catalyst preparation}

Chicken eggshells were collected from nearby hotels. Collected eggshells were washed several times to remove impurities for the calcination process. Washed eggshells were dried in sunlight for long times. It was then crushed in a grinder to make a fine powder. This powder was taken to the muffle furnace for the calcination process. Eggshell powder calcined at $900^{\circ} \mathrm{C}$ for 4 hours in the muffle furnace. Above $800^{\circ} \mathrm{C}$, Calcium Carbonate is decomposed and converted as Calcium Oxide. This calcium oxide stored in an air-tight container for future use [14].

The chemical equation for transformation of chicken eggshells to catalyst as follows:

$\mathrm{CaCO}_{3}(s) \stackrel{900 \mathrm{C}}{\longrightarrow} \mathrm{CaO}(s)+\mathrm{CO}_{2}$

\subsection{Catalyst characterization}

The morphology and elemental chemical contents of the synthesized catalysts before calcination and after calcination were measured by field emission scanning electron microscopy (FESEM). The X-ray diffraction (XRD) analysis was performed at room temperature. $\mathrm{Cu} k \alpha$ radiation $(\lambda=$ $0.15406 \mathrm{~nm}$ ) in a $2 \theta$ scan range of $10-90^{\circ}$ was used for all samples.

\subsection{Taguchi experimental design}

Geneichi Taguchi designed and developed the best tool for an effective quality process or system. Experimental design and quality loss function can be analyzed through the effective design of experiments with the Taguchi tool. This is a tool for optimizing the process parameters with less trial. Taguchi orthogonal array is a cost-effective and time savings technique for optimizing the experimental process parameters. In this study, three factors have selected namely methanol to oil molar ratio, reaction temperature and catalyst loading in the transesterification process. Input parameters and their levels are tabulated in Table 2. In the Taguchi method, the orthogonal array is selected based on degrees of freedom (DOF) of process parameters. Total input parameters of DOF should be less than the selected DOF of the orthogonal array. DOF of individual parameter is subtracting one from several levels of parameters that are shown in Table 2. Three DOF factors with three levels are 6; hence L9 orthogonal array is selected [14]. DOF of L9 orthogonal array is 8; this is higher than the total DOF input parameters. Henceforth, this design accommodates all factors for investigation of transesterification parameters of bio-fuel yield and its viscosity.

\subsection{Transesterification process procedure}

Linseed oil was used in the transesterification process with the help of $250 \mathrm{ml}$ two-necked flask, a condenser and a magnetic stirrer in a water bath. Particular amount of linseed oil, methanol and $\mathrm{CaO}$ catalyst are introduced in the reaction flask. A magnetic stirrer was continuously stirred during entire reaction. Particularly, for optimum bio-fuel yield, oil to methanol molar ratio of $1: 8$, reaction temperature $60^{\circ} \mathrm{C}$, reaction time $1.3 \mathrm{~h}$, catalyst loading $6 \% \mathrm{w} / \mathrm{w}$ were used. 600 rpm stirring speed was used for all the experiments. The transesterification process experimental arrangements was shown in Fig. 1. After the reaction, transesterified oil is transferred to separation funnel and left for $24 \mathrm{~h}$ undisturbed to separate glycerol and bio-fuel. Glycerol is a byproduct from this reaction and bio-fuel is taken for several wash with the help of warm distilled water to remove un-reacted catalyst. Table 3 shows L9 orthogonal array was used in this study including input parameters of transesterification process with corresponding output response. 


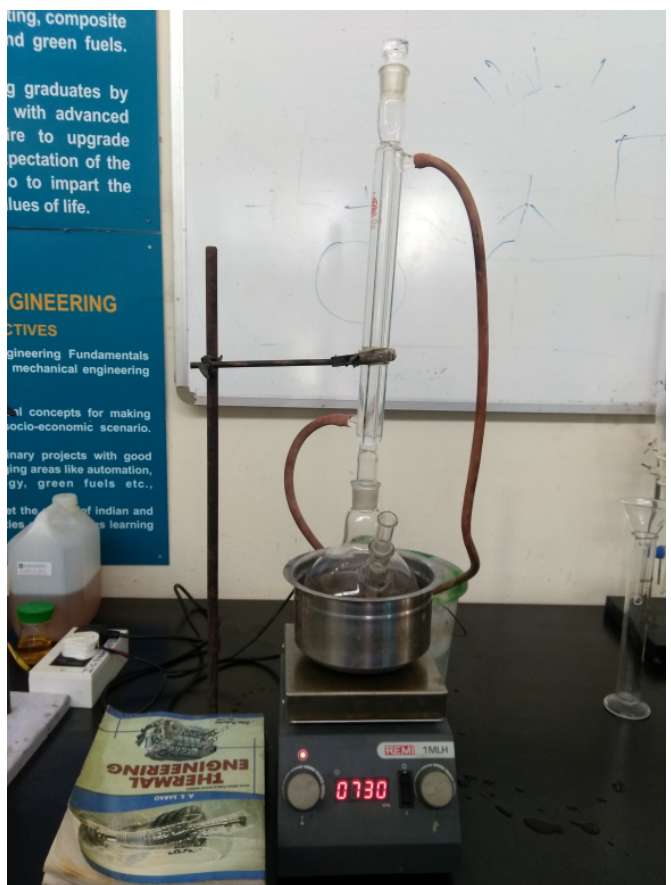

Fig. 1. Transesterification Process Arrangements

\subsection{Grey Relational Analysis method}

GRA is a multi-objective decision-making technique used in all engineering fields as its simplicity and it takes less time to evaluate the responses. The GRA technique was established by Deng in 1982. GRA used to analyze the uncertainties of the system. In this GRA approach, all output responses are normalized between 0 and 1 . Normalization is to easily interpret the data and analysis. The normalized values are used to calculate the Grey Relational Coefficient (GRC) from all output data. Grey Relational Grade (GRG) is calculated by averaging GRC. The performance of the entire experimental trail highly depends on Grey Relational Grade. A higher in the GRG value gives an optimum solution towards the response. The following procedure was used in GRA techniques.

Step I: Normalization of entire experimental data

As per the required conditions, normalization for higher-thebetter

$x_{i}(k)=\frac{v_{i}(k)-\min v_{i}(k)}{\max v_{i}(k)-\min v_{i}(k)}$

Normalization for lower-the-better

$x_{i}(k)=\frac{\max v_{i}(k)-v_{i}(k)}{\max v_{i}(k)-\min v_{i}(k)}$

where, $v_{i}(k)$ is the normalized value for experimental output response; $\min v_{i}(k)$ is the minimum value of $v_{i}(k)$ for $\mathrm{k}^{\text {th }}$ response; $\max v_{i}(k)$ is the maximum value of $v_{i}(k)$ for $\mathrm{k}^{\text {th }}$ response.

Step II: Calculation of Grey Relational Coefficient

It is calculated to create correlation between ideal and actual normalized values.

$\gamma_{i}(k)=\frac{\Delta_{\min }+\omega \Delta_{\max }}{\Delta_{o i}(k)+\omega \Delta_{\max }}$ where, $\quad \Delta_{o i}(k)=\left|x_{0}(k)-x_{i}(k)\right|, \omega$ is the distinctive coefficient, which is used to enlarge or compress the range of grey relation coefficient. The value of $\omega$ is between 0 and 1 . The value for $\omega=0.5$ is preferable [15]. $\Delta_{\min }$ is minimum of $\Delta_{o i}$ and $\Delta_{\max }$ is maximum value of $\Delta_{o i}$.

Step III: Calculation of Grey Relational Grade (GRG)

$\delta_{i}=\frac{1}{n} \sum_{i=1}^{n} \gamma_{i}(k)$

where, $\delta_{i}$ is grey relational grade, $\mathrm{n}$ is number of output response

\section{Result and Discussion}

\subsection{Characterization of feedstock (\%FFA)}

The characterization of the linseed oil is shown in Table 1. Linseed oil contains $2.4 \%$ of FFA, which is suitable for the alkali based transesterification process. If any feedstock is contained either higher acid value or FFA content, which certainly produces low TG in the transesterification reaction. Rice-bran oil contains high FFA, which produces a low conversion of TG during the transesterification process. Castor-oil also contains high FFA content, but which is not produced any TG during the transesterification process [16]. Acid-based transesterification or alkali based transesterification is chosen based on the acid value and FFA. Miss selection of catalysts in the transesterification process not only leads to cost factor but also time factors.

\subsection{Grey Relational Analysis}

The objective of this study, investigate the transesterification process parameters on yield and viscosity of linseed oil for the bio-fuel production. Output responses of transesterification process are normalized using equation (2) considering higherthe-better. Similarly, experimental responses are normalized using equation (3) considering lower-the-better. Normalized value of output responses, GRC and GRG are calculated using equation (2), (3), (4) and (5) and are tabulated in the Table 4. The value of distinctive coefficient $\omega$ is taken as 0.5 and Grey Relational Coefficient are calculated. In the Grey Relational Analysis, GRG values near to 1 are taken as optimum solution. The experimental trial 6 has a high GRG value of 0.8 among the 9 experiments; this conveys that experimental trial 6 provides better bio-fuel yield and viscosity. The molar ratio of $8: 1$, reaction temperature $60{ }^{\circ} \mathrm{C}$ and catalyst loading of $7 \% \mathrm{w} / \mathrm{w}$ gives good results among the selected input transesterification parameters.

The mean Grey Relational Grade (MGRG) of entire level of experimental process parameters was calculated by taking the average of Grey Relational Coefficient at the same factor level was shown in the table (5). The effect of transesterification parameters on MGRG was shown in Fig. 2. By means of chart, optimal process parameter for transesterification of linseed oil for high biodiesel yield and low viscosity was recognized as MR3RT3CL3, which are methanol to oil molar ratio of $8: 1$, reaction temperature of 65 ${ }^{\circ} \mathrm{C}$ and catalyst loading of $7 \% \mathrm{w} / \mathrm{w}$. 

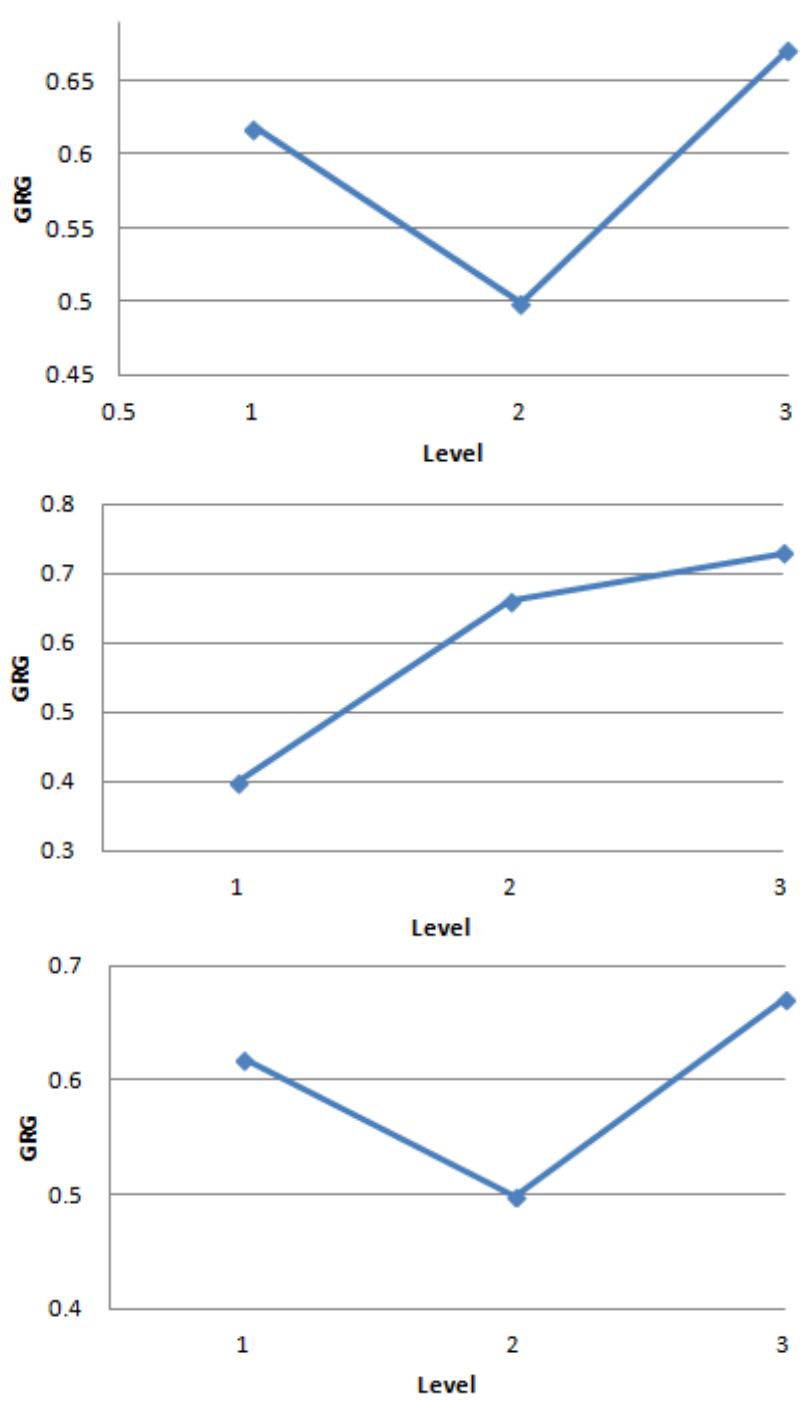

Fig. 2. Effect of Transesterification process parameters on GRG

\subsection{Characterization of catalyst}

XRD spectra of uncalcined and calcined eggshells sample was obtained with $\mathrm{Cu}$ radiation at $45 \mathrm{kV}, 30 \mathrm{~mA}$ and a scan range of $10-80^{\circ}$. The XRD profiles of raw eggshell and calcined eggshell are shown in Fig. 2. For the raw eggshell (Fig. 3 a), the most intense diffraction peak was seen at $2 \theta=$ $29.36^{\circ}$ with height of 2683.97 cts. The other typical peaks were noticed $31.38^{\circ}, 35.56^{\circ}, 39.35^{\circ}, 43.07^{\circ}$. These peaks matched precisely with reported by [14]. These peaks were characteristic of calcium carbonate. For the calcined catalyst as showed in Fig. $3 \mathrm{~b}$, the peaks were appeared at $2 \theta=$ $18.2681,2 \theta=23.1808,2 \theta=29.4704,2 \theta=34.2237,2 \theta=$ $39.5250,2 \theta=47.6788,2 \theta=57.5379$, which were characteristics of calcium oxide. However, it was further observed that the presence of calcium hydroxide at $2 \theta=$ 18.2681. The XRD pattern of the prepared waste eggshells sample calcined at $900{ }^{\circ} \mathrm{C}$ exhibited similar results to [11]



a)

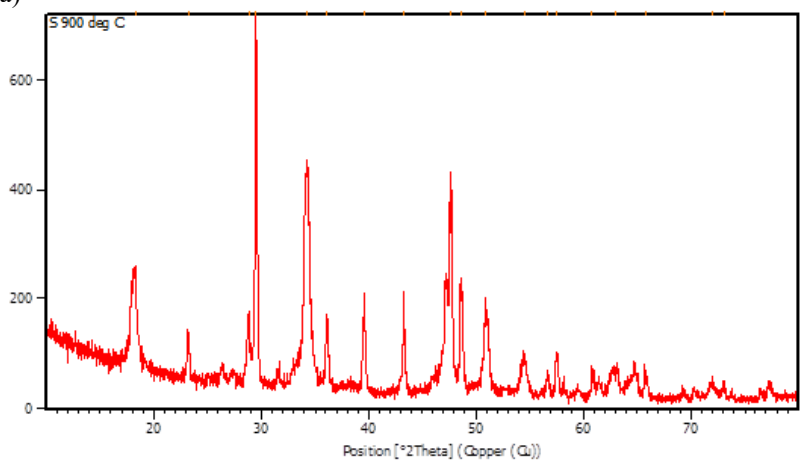

b)

Fig. 3. a. XRD Pattern of waste chicken eggshells before calcination and $b$. XRD Pattern of waste chicken eggshells after calcination

\section{Conclusion}

In this proposed study, waste material namely waste eggshells converted into catalyst for the bio-fuel production. The waste eggshells were calcined at $900^{\circ}$. The characteristics of uncalcined and calcined eggshells were studied with the help of XRD. Uncalcined eggshells namely raw eggshells contained $\mathrm{CaCO}_{3}$ and upon calcination it was converted to $\mathrm{CaO}$. This $\mathrm{CaO}$ effectively used in the bio-fuel synthesis. Linseed oil was selected for this study as considering its abundant availability. Alkali catalyst transesterification was done as this linseed oil contains low FFA content. Taguchi orthogonal array was formed and L9 array was selected in this study. Nine alkali catalyst based transesterification was conducted and its yield as well as viscosity was noted. GRA multi-objective decision-making approach was used to optimize the conducted experiments. In this regard, experimental trial 6 has the highest Grey Relational Coefficient value 0.8 and it was ranked first position. The input transesterification parameters for experimental trial 6 was methanol to oil molar ratio of $8: 1$, reaction temperature of $60^{\circ} \mathrm{C}$ and catalyst loading of $7 \% \mathrm{w} / \mathrm{w}$. The mean Grey Relational Grade of entire level of experimental process parameters was calculated. Based on the closeness coefficient, transesterification of linseed oil with eggshells derived catalyst for producing high bio-fuel yield and low viscosity was recognized as MR3RT3CL3, which are methanol to oil molar ratio of $8: 1$, a reaction temperature of $65^{\circ} \mathrm{C}$ and catalyst loading of $7 \% \mathrm{w} / \mathrm{w}$.

This is an Open Access article distributed under the terms of the Creative Commons Attribution License 


\section{References}

[1] A. Sandouqa and Z. Al-hamamre, "Energy Analysis of Biodiesel production from Jojoba Seed Oil,” Renew. Energy, 2018.

[2] P. Singh, S. K. Tiwari, R. Singh, and N. Kumar, "Modi fi cation in combustion chamber geometry of CI engines for suitability of biodiesel : A review," vol. 79, no. May, pp. 1016-1033, 2017.

[3] V. Karthickeyan, "Effect of cetane enhancer on Moringa oleifera biodiesel in a thermal coated direct injection diesel engine," Fuel, vol. 235, no. August 2018, pp. 538-550, 2019.

[4] P. R. Pandit and M. H. Fulekar, "Egg shell waste as heterogeneous nanocatalyst for biodiesel production: Optimized by response surface methodology," J. Environ. Manage., vol. 198, pp. 319-329, 2017.

[5] N. Mansir, S. Hwa, M. L. Ibrahim, and Y. Hin, "Synthesis and application of waste egg shell derived $\mathrm{CaO}$ supported W-Mo mixed oxide catalysts for FAME production from waste cooking oil : E ff ect of stoichiometry," vol. 151, no. August, pp. 216-226, 2017.

[6] Y. Hua, M. Omar, and C. Nolasco-hipolito, "The potential of waste cooking oil-based biodiesel using heterogeneous catalyst derived from various calcined eggshells coupled with an emulsi fi cation technique: A review on the emission reduction and engine performance," Renew. Sustain. Energy Rev., vol. 47, pp. 589-603, 2015.

[7] P. R. Pandit and M. H. Fulekar, "Biodiesel production from microalgal biomass using $\mathrm{CaO}$ catalyst synthesized from natural waste material," Renew. Energy, 2019.

[8] Y. H. Tan, M. O. Abdullah, C. Nolasco-hipolito, and N. A. Zauzi, "Application of RSM and Taguchi methods for optimizing the transesterification of waste cooking oil catalyzed by solid ostrich and chicken-eggshell derived CaO," Renew. Energy, 2017.

\section{Nomenclature}

$\begin{array}{ll}\mathrm{CaO} & \text { - Calcium Oxide } \\ \mathrm{CaCO}_{3} & \text { - Calcium Carbonate } \\ \text { TOPSIS } & \text { - Technique for Order Preference by Similarity to Ideal Solution } \\ \text { FAME } & \text { - Fatty Acid Methyl Ester } \\ \mathrm{FAA} & \text { - Free Fatty Acid } \\ \mathrm{XRD} & \text { - X ray Diffraction } \\ \mathrm{MR} & \text { - Molar Ration } \\ \mathrm{RT} & \text { - Reaction Temperature } \\ \mathrm{CL} & \text { - Catalyst Loading } \\ \mathrm{T}_{\mathrm{m}} & \text { - TOPSIS Design matrix } \\ \mu_{\mathrm{ij}} & \text { - The normalized value of the design matrix } \\ \mathrm{S}_{\mathrm{i}}^{+} & \text {- Positive Separation measures } \\ \mathrm{S}_{\mathrm{i}}^{-} & \text {- Negative Separation measures } \\ \tau_{\mathrm{ij}} & \text { - Weighted normalized matrix } \\ \mathrm{CC} & \text { - Closeness Coefficient } \\ \tau^{-} & \text {- Negative ideal solution } \\ \tau^{+} & \text {- Positive ideal solution } \\ \mathrm{MCC} & \text { - Mean Closeness Coefficient }\end{array}$

[9] P. Ferraz and S. Meireles, "Highly active $\mathrm{CaO}$ catalysts from waste shells of egg and clam for biodiesel production," "Applied Catal. A, Gen., 2018.

[10] M. Kirubakaran and A. M. S. V, "Eggshell as heterogeneous catalyst for synthesis of biodiesel from high free fatty acid chicken fat and its working characteristics on a CI engine," Biochem. Pharmacol., 2018.

[11]K. Rahees and V. Meera, "Production of Biodiesel from Dairy Waste Scum,” Int. J. Sci. Eng. Res., vol. 5, no. 7, pp. 194-199, 2014.

[12] M. Farooq et al., "Biodiesel production from date seed oil (Phoenix dactylifera L.) via egg shell derived heterogeneous catalyst," Chem. Eng. Res. Des., vol. 132, pp. 644-651, 2018.

[13]P. Karthikeyan, P. Lokesh, and P. Suneel, "Performance and emission characteristics of direct injection diesel engine using linseed oil as biodiesel by varying injection timing," Int. J. Ambient Energy, vol. 40, no. 1, pp. 35-39, 2019.

[14]A. R. Gupta and V. K. Rathod, "Waste cooking oil and waste chicken eggshells derived solid base catalyst for the biodiesel production: Optimization and kinetics," Waste Manag., vol. 79, pp. 169-178, 2018.

[15]P. Baranitharan, K. Ramesh, and R. Sakthivel, "Multi-attribute decision-making approach for Aegle marmelos pyrolysis process using TOPSIS and Grey Relational Analysis: Assessment of engine emissions through novel Infrared thermography," J. Clean. Prod., 2019.

[16]R. Kumar, P. Tiwari, and S. Garg, "Alkali transesterification of linseed oil for biodiesel production," Fuel, vol. 104, pp. 553-560, 2013. 